\title{
Sonia Combe, La loyauté à tout prix. Les floués du „socialisme réel“", Lormont, les éditions Le Bord de l'eau, 2019
}

\author{
Compte rendu / Rezension / Review
}

Dorothee Röseberg

Leibniz Sozietät der Wissenschaften zu Berlin / Martin-Luther-Universität Halle-Wittenberg

Die bekannte DDR- und Osteuropaexpertin unter den französischen Historikerinnen, Sonia Combe, legt mit ihrem im Herbst 2019 erschienenen Buch einen bemerkenswerten Beitrag zur DDR-Intellektuellengeschichtsschreibung vor. Das Originelle besteht zunächst darin, dass der Blick vergleichend auch auf andere osteuropäische Staaten gerichtet ist. Das Cover zeigt Anna Seghers mit Georg Lukács 1952 in Budapest als Hinweis auf eine enge Verbundenheit dieser Intellektuellen untereinander, obgleich sich deren Wege später mitunter auch trennten.

Das Interesse der Historikerin gilt der Gründergeneration in diesen Ländern, insbesondere in der DDR und hier den Wissenschaftlern, Schriftstellern und Künstlern, die aus marxistischer und kommunistischer Überzeugung - nach Vertreibung und Exil - die SBZ bzw. die DDR als ihre ideologische, politische und kulturelle Heimat gewählt hatten. Sie waren ob ihrer politischen Überzeugungen und meist wegen ihrer jüdischen Herkunft im Dritten Reich verfolgt worden und hatten Deutschland verlassen müssen. Viele von ihnen waren nach Frankreich, Spanien, Süd-, Mittel- oder Nordamerika geflohen, was innen bei ihrer Rückkehr den Status von „Westemigranten“ einbrachte. Der Konflikt zwischen diesen Intellektuellen als Rückkehrer „aus dem Westen“ und jenen Remigranten, die aus dem Osten, aus Moskau kamen, ebenfalls kommunistisch überzeugt, politisch verfolgt und vielfach jüdischer Herkunft, die dort ihr Exil gesucht hatten, gehört zu den zentralen Problemlagen der DDR-Geschichte. Beide, Ost- wie Westremigranten konstituierten die Gründergeneration in der DDR, die trotz Besatzungsstatut über die 40 Jahre der Existenz der DDR hinweg eine wesentliche Rolle gespielt hat, nicht zuletzt, weil sie der Folgegeneration durch ihre Biografien als Verfolgte des Naziregimes als moralische Instanzen dienten. Walter Ulbricht und Wilhelm
Pieck stehen für die Gruppe der Moskau-Rückkehrer, die die obersten Ränge der politischen Funktionen, nicht nur in den Anfangsjahren, innehatten. Westemigranten waren hingegen von der höchsten parteipolitischen Verantwortung in der DDR weithin ausgeschlossen. Sie hatten mit Misstrauen, Verdächtigungen, sogar politischen Prozessen zu kämpfen. Doch sie blieben - bis auf einige Ausnahmen - der DDR treu. Von ihnen ist bei Sonia Combe hauptsächlich die Rede. Zu innen gehören auch über die Grenzen der DDR hinaus bekannte Wissenschaftler, Schriftsteller und Künstler: Anna Seghers, Bertolt Brecht, Ernst Busch, Arnold Zweig, Ernst Bloch, u.v.a. wie auch Jürgen Kuczynski, dem ein gesondertes Kapitel gewidmet ist. In den ersten Dezennien des 20. Jahrhunderts geboren oder/und ihre Kindheit in dieser Zeit verbracht, bildeten sie eine „Erfahrungsgeneration“, die charismatische Persönlichkeiten hervorbrachte, die schon bei ihrer Rückkehr in die SBZ/DDR als Autoritäten anerkannt waren.

Titel und Untertitel der Studie von Sonia Combe lassen bereits das Programm erahnen, dem sich die Autorin widmet. Es geht um Spannungen und Konflikte, die diese Intellektuellen im Verlauf ihres Lebens in der DDR erlebt haben, um ihre „Loyalität um jeden Preis" der Partei und dem politischen System gegenüber und um ihre Erfahrungen, die sie letztlich zu „Betrogenen durch den Realsozialismus“ hat werden lassen; Betrogene als kritische Marxisten, denen es um einen Sozialismus bzw. Kommunismus ging, dem der reale Sozialismus immer weniger gerecht wurde.

Im Zentrum der Arbeit von Sonia Combe stehen intellektuelle Akteure $^{1}$, die das System der DDR einerseits stützten und es zugleich in Frage stellten. Es handelt sich um eine

1 Bei den Kursivierungen handelt es sich um Hervorhebungen der Rezensentin. 
differenzierte Sicht auf die DDR-Intellektuellengeschichte, die eine ebenso differenzierte Lektüre verlangt. ${ }^{2}$

Teil I des Buches widmet sich mit dem Titel „Hoffnung“ der Rückkehr dieser Akteure nach Deutschland. Die Analyse von Sonia Combe ist darauf gerichtet, konkret an vielen Beispielen, mit Dokumenten, oft auch aus erst kürzlich zugänglichen Archiven, zunächst den Gründen nachzugehen, warum die Wahl auf die SBZ/DDR fiel. Marxistische Überzeugungen, der Glaube (ein Begriff, den die Autorin bewusst ausspart) an die Realisierung einer kommunistischen Gesellschaftsordnung, zeigen sich gepaart mit pragmatischen und real politischen Gegebenheiten: So rangen die SBZ und die junge DDR um die Unterstützung dieser Intellektuellen durch eine Kulturpolitik, die für die Remigranten Wohnungen (u.a. das berühmte Intelligenzviertel in Berlin Pankow am Majakowski Ring), Arbeit und gute Entlohnung wie symbolische Ehrungen einschloss (Berufungen an Universitäten, Nationalpreise etc.), sowie auch ein Netzwerk und Institutionen, wo diesen Akteuren ein Handlungsfeld ermöglicht wurde (z.B. „Die Möwe" in der Berliner Luisenstraße als berühmter Treff von Künstlern). Enttäuschungen und Verzweiflung einiger der Rückkehrer und ihrer Begleitung werden ebenfalls belegt.

Teil II des Buches zeichnet die „Entzauberung“ nach. Es ist vielleicht der wichtigste Teil des Buches. Denn hier werden die Gründe für den zunehmenden Dissens zwischen den Intellektuellen und der „bestimmenden Parteilinie“ sowie deren Folgen dokumentiert. Anlässe sind die Konflikte auslösenden historischen Ereignisse wie der Juniaufstand 1953, Ungarn 1956, der Tod Stalins, Prag 1968 etc. In dieser Phase erwerben diese Intellektuellen das Attribut „kritische“ Intellektuelle zu sein, wovon „die Öffentlichkeit“ allerdings so gut wie nichts erfährt. Denn sie schweigen. Das Schweigen wird eine dominante Haltung jener Intellektuellen, die ob ihrer öffentlichen Ausstrahlung doch hätten das Wort ergreifen können/ sollen? Und sie taten es, wie die Autorin anhand von bislang nur wenig erschlossenen Dokumenten, darunter des Schriftstellerverbandes, nachweist, aber nur innerhalb der Partei.

Das Schweigen als „Antwort“ auf die Erfahrungen im „realen Sozialismus" ist ein Kompromiss - so Sonia Combe -, der allerdings stets die Gefahr der Kompromittierung in sich trug. Schweigen als Kompromiss, um nicht Verrat an der Idee des Sozialismus/Kommunismus zu üben oder den „Gegnern“ Argumente in die Hand zu geben, aber auch um nicht zu lügen. Wenn das Schweigen nur innerhalb der Partei gebrochen wurde und dort selbst Debatten um einen demokratischen Sozialismus geführt worden sind, dann sei dies, so die Autorin - einem irrationalen Glauben an die Reformfähigkeit der Partei von innen heraus geschuldet. Darin sieht Sonia Combe das Scheitern dieser Intellektuellen begründet (S. 223). In jedem Fall ist das Schweigen ein Verhaltensmodus, der dem historischen Typus des engagierten Intellektuellen nicht entspricht, anders als es die Autorin zumindest an einer Stelle ihrer Studie (S. 23) aussagt. Man könnte

2 Wenn Jürg Altwegg in der FAZ vom 28. Februar 2020 (S. 10) seine Rezension unter das Motto einer "Ostalgie à la française" stellt, so schließt er sich selbst aus dem Kreis der Leser aus, die dieses Buch auf eine differenzierte Weise gelesen haben. allerdings dieses Schweigen als ein „beredtes Schweigen“ qualifizieren. Dem kommt auch das Kapitel von Sonia Combe entgegen, das am Schluss ihres Buches den Titel „La performance théâtrale" trägt. Beredt sind z.B. Lächeln und Handschlag von Anna Seghers: Sie verlässt den Raum nach einer Diskussion, in der das Stück von Heiner Müller „Die Emigrantin“ unter das Verdikt des Verbotes gefallen war; sie hatte geschwiegen, aber sie reichte im Hinausgehen Heiner Müller lächelnd und ostentativ die Hand (S. 220).

Das Schweigen ist somit das eigentliche Thema des Buches von Sonia Combe. Man mag sich als Leser fragen, warum die Autorin das Schweigen nicht im Titel als zentrales Thema anführt. Schließlich durchzieht das Schweigen mehrere Kapitel. Zentral ist dieses Thema in der Einleitung zum zweiten Teil des Buches platziert. Mehr noch, denn dieses Schweigen ist für Sonia Combe nicht nur eine Haltung, sondern vielmehr eine Éthique $d u$ silence und damit ein moralisches Gebot für diese Intellektuellen. Unter der Überschrift "Ethik des Schweigens" formuliert die Autorin einige Kernaussagen ihrer Studie, die um das Thema „Schweigen“ kreisen: „Die kommunistischen osteuropäischen Staaten wurden auf der Grundlage des Schweigens aufgebaut" (S. 88). Sonia Combe meint damit das Schweigen über die Verbrechen Stalins, über den Hitler-Stalin-Pakt 1939, über das „Verschwinden" von deutschen Kommunisten vor und während des Krieges in den Lagern der Sowjetunion. Und geschwiegen wurde über die antisemitische Welle, die unmittelbar nach dem Krieg von der UdSSR ausging, über die Schauprozesse in Budapest, Prag und Warschau und auch über die Repressalien gegenüber den Spanienkämpfern und anderen Westemigranten. Spätestens an dieser Stelle wird klar, dass das Schweigen der Gründergeneration in der DDR, insbesondere der Westemigranten unter innen, nicht einfach ein selbstauferlegtes, „freiwilliges" Schweigen war. Sonia Combe beschreibt eindringlich, wie nicht nur die Stalinanhänger in der Sowjetunion, sondern auch deutsche Moskau-Remigranten einen Habitus verinnerlicht hatten, der eine Mischung aus Misstrauen und aus Erwartung einer absoluten und bedingungslosen Gefolgschaft war, einer Gefolgschaft gegenüber der Moskauer Parteilinie. Die erwartete Geheimhaltung stellte eine mächtige Kontrollmodalität dar und zog das Schweigen nach sich. Gerechtfertigt wurde das Schweigen im Namen der Rettung des kommunistischen Ideals und - so Sonia Combe - das Schweigen war keine Geste der Unterwerfung, sondern eine „Art zweite Natur", eine angenommene Disposition, die nicht mehr hinterfragt wurde. Diese Haltung änderte sich - anders als bei vielen Kommunisten in den westeuropäischen Staaten, die nach dem 20. Parteitag der KPdSU die kommunistischen Parteien verließen - im Osten nur wenig. Dies gilt auch für die Folgegeneration, zu der Christa Wolf, Heiner Müller u.a. gehörten, denen sich Sonia Combe im Teil III widmet. Dennoch stellt die Autorin einen Unterschied fest, wenn sie davon ausgeht, dass das Schweigen in der DDR, im Vergleich zu anderen osteuropäischen Gesellschaften, ganz besonders respektiert worden sei. In der Konsequenz habe es deshalb in der DDR auch keine frühe organisierte 
Dissidentenbewegung gegeben wie etwa in Polen, Ungarn oder in der Tschechoslowakei.

Mit dem Thema des Schweigens erfasst die Autorin ein Grundproblem, das viele Intellektuelle, auch diejenigen der Folgegenerationen, nach 1989 beschäftigt hat. Wenn der Aufruf „Für unser Land“, maßgeblich von Christa Wolf am 26. November 1989 initiiert, und von vielen der kritischen Intellektuellen unterzeichnet und somit eine öffentliche Stimme war, kam diese jedoch zu spät, um das Land eigenständig und auf einen Reformweg des Sozialismus zu führen und zu orientieren.

Wenn es im Grunde um dieses Schweigen geht, dann darf die Frage danach gestellt werden, welche Erwartungen der Titel des Buches suggeriert: Wird hier nicht im Verbund von Bild und Schrift auf das Leben einer Generation von Schriftstellern und Wissenschaftlern rekurriert, das sich nun zweifellos und dem würde Sonia Combe sicher zustimmen - nicht auf das Schweigen reduziert? In und mit ihren Werken, die in der DDR breit rezipiert worden sind, haben sie ein intellektuelles Leben bestimmt, das sich gerade auch an innen orientiert hat. Die stets vergriffenen Auflagen vor allem dieser Schriftsteller, einschließlich der „Erben“, sind ein Beleg für das breite Echo, das sie in verschiedenen Schichten der DDR-Bevölkerung gefunden haben. Sonia Combe weist am Rande darauf hin, wie auch auf die Praxis des „Zwischen den Zeilen Lesens", bei dem ein stiller Konsens darüber bestand, dass vor allem in der Literatur und Kunst Debatten initiiert und ausgetragen wurden, die in der Presse und Politik zum Schweigen verurteilt waren. Diese Generationen waren nicht nur „betrogen“ worden, sondern sie haben ganz wesentlich in der Lebenswelt und den Lebensweisen der meisten DDR-Bürger ein Echo gefunden; ein Echo, das sich, wie die britische Historikerin Mary Fulbrook schreibt, als ,Ein als Ob' beschreiben lässt und das damit auf die verschiedenen Praktiken zwischen Anpassung und Widerstand, sowie auf eine Alltagssphäre verweist, „die aus einem komplexen Geflecht von Mikrobeziehungen der Macht" bestand (Fullbrook 2008: 252). Auch die Gesten des „beredten Schweigens“ gehören zu diesen Mikrobeziehungen der Macht. Außerdem waren die bekannten Nischen in der DDR Orte, um solche Diskussionen weiterzuführen.

Dessen ungeachtet ist das Buch von Sonia Combe wichtig: Es zeigt an vielen gut dokumentierten Beispielen, dass der Antifaschismus in der DDR keineswegs nur ein Mythos war, wie dies oftmals behauptet wird, sondern eine gelebte Erfahrung und Haltung mit moralischem Anspruch. Die Gründergeneration steht für diese Haltung, die mit einem moralischen Impetus an die Folgegenerationen vermittelt worden ist. Das Buch von Sonia Combe zeigt außerdem an konkreten Debatten, dass die SED keineswegs ein homogener Block war, sondern dass sie sich - vor allem seit den 70er, verstärkt in den 1980er Jahren - zu einem Ort kritischer Auseinandersetzungen entwickelt hatte. Der Leser mag verstehen, wie für die Älteren ihre Erfahrungen der Repression und für alle ein von der Stasi durchzogenes System, den Handlungsradius so vieler Intellektueller bestimmte und einengte. Deshalb könnte man das Fazit formulieren: Die DDR ist auch an einem oktroyierten Schweigen gescheitert.

Hinzu kommt, dass Sonia Combe in den essayistisch gehaltenen Teilen ihres Buches einige Hypothesen formuliert, die zur weiteren Diskussion herausfordern, mit denen die Autorin auf tiefer liegende Erklärungszusammenhänge für die Loyalität dieser Intellektuellen rekurrieren möchte. Sie führen sie zu einem Teil deutscher-jüdischer Geschichte.

Aus dem Befund, dass die Gründergeneration der DDR zahlreiche Juden in ihren Reihen hatte, auch gerade unter den kritischen Intellektuellen, interessiert sich Sonia Combe für die sogenannte „deutsch-jüdische Symbiose“ (S. 195). Zu den Eigentümlichkeiten der Intellektuellen jener Generation in der DDR gehörte, dass die jüdischen Wurzeln weniger im Vordergrund standen als die kommunistische Überzeugung. Dennoch misst die Autorin diesen jüdischen Wurzeln eine Bedeutung bei, wenn sie davon ausgeht, dass sich der im Judentum verbreitete Messianismus auf eine besondere Weise mit dem Kosmopolitismus des Kommunismus trifft, verbindet und in der DDR auch den Hintergrund für die Loyalität der kritischen Intellektuellen gegenüber der Partei und dem System bildete. Sie sahen sich in der DDR, trotz Verfolgung und Exil, weniger als Opfer, denn als Kämpfer für eine bessere, sozialistische Welt. Dies verband sie im Übrigen mit den Ost-Remigranten. Sonia Combe übernimmt den Begriff der deutsch-jüdischen Symbiose von Tucholsky, der ihn auf die Zeit der Weimarer Republik angewandt hatte. Man kann sich aber fragen, ob und wie der messianische Glaube wirklich „präsent“ bei jenen war, die ihre jüdischen Wurzeln in der DDR oftmals kaum wahrgenommen, geschweige denn gepflegt hatten? Denn viele jener jüdischen Kommunisten waren bereits in jüdisch assimilierten Familien aufgewachsen. Wie auch immer, in der DDR wollten sie ebenso assimiliert und nicht als Minderheit oder Außenseiter gelten. Vielmehr sahen sie sich in ihrem „Kampf“ um ihre Utopie als Teil einer Mehrheitsgesellschaft. Die Loyalität gegenüber der kommunistischen Partei, die ein „beredtes Schweigen“ einschloss, das jene Intellektuellen nicht selten als Momente ihres "Widerstands" angesehen haben mögen, all dies sieht Sonia Combe auch im Kontext dieser "deutsch-jüdischen Symbiose“, die in der DDR eine „letzte“ Verwirklichung gefunden hätte. Das bietet Anlass für weitere Debatten.

Das Verdienst von Sonia Combe ist es, einen überzeugenden Beitrag zu einer differenzierteren Sicht auf die DDR-Intellektuellengeschichte, einschließlich einer Diskussion darüber, geleistet zu haben. Mit der Nachzeichnung der Biographien und Haltungen charismatischer Persönlichkeiten aus Kunst, Wissenschaft und Kultur wird deutlich, wie vielfältig und plural die Überzeugungen vom Sozialismus in der DDR waren und wie sie sich in der Gründergeneration aus den Erfahrungen mit dem Dritten Reich generierten. Die Autorin lehnt zu Recht eine Gleichsetzung der beiden Diktaturen ab.

Einig mag man mit der Autorin in der Frage der Folgen des Schweigens sein, zumindest liegt es nahe, dass dieses Schweigen längere Zeit zur Stabilisierung des Systems beigetragen hat. 
Sonia Combes Buch ist ein Argument gegen eine Historiographie, die die Geschichte der "real-sozialistischen“ Gesellschaften im 20. Jahrhundert auf eine einfache Diktaturgeschichte reduziert. Es ist ein Buch über einen Teil deutscher und europäischer Geschichte, welches das 20. Jahrhundert auch als ein Jahrhundert gelebter Utopien ausleuchtet und dies mit ihren Herausforderungen, Widersprüchen und ihrem
Scheitern. Es mag auch eine Einladung sein, um die Intellektuellengeschichte der DDR und der osteuropäischen Staaten auf differenzierte Weise weiterzuschreiben.

Eine Übersetzung ins Deutsche ist zu empfehlen, nicht zuletzt auch für die Generationen der "Nachwendekinder“ in einem weiten, d.h. gesamtdeutschen und europäischen Sinne: Ihnen dürfte das Zeitalter der großen Ideologien fremd sein.

\section{Bibliographie}

Altwegg, Jürg, „Auch Plastikblumen wecken Erinnerungen“, in Frankfurter Allgemeine Zeitung, 28.2.2020.

Fulbrook, Mary (2008), Ein ganz normales Leben. Alltag und Gesellschaft in der DDR, Darmstadt, Primus Verlag. 